\title{
Salvage Resection of Mediastinal Nonseminomatous Germ Cell Tumor in a Patient with Extrathoracic Involvement upon Progression following High-Dose Chemotherapy
}

\author{
Jia-Ling Chou ${ }^{a}$ David Tse ${ }^{b}$ \\ aMedical Oncology, Southern California Permanente Medical Group, Woodland Hills, CA, USA; \\ bThoracic Surgery, Northwest Permanente, Mt Talbert Medical Office, Clackamas, OR, USA
}

\section{Keywords}

Mediastinal mass · Germ cell tumor · High-dose chemotherapy · Salvage resection

\begin{abstract}
Primary mediastinal nonseminomatous germ cell tumor with extrathoracic metastases is associated with a very high mortality rate, and there is no consensus regarding optimal upfront therapy. Once patients fail the first-line treatment, salvage therapy often fails to effectively control the disease. Resection of the residual mediastinal mass does not appear to achieve long-term control in those who have extrathoracic metastases following conventional firstline systemic therapy. We report a case where a young man presented with symptomatic brain metastases as well as extensive visceral involvement of the liver, small intestine, and lungs. He was successfully managed with multimodality treatment including high-dose chemotherapy with hematopoietic stem cell support following standard first-line chemotherapy, resection of mediastinal disease, lung metastasectomy, and stereotactic brain radiation. He has achieved long-term survival.
\end{abstract}

\section{Introduction}

Primary mediastinal nonseminomatous germ cell tumor (NSGCT) is rare and falls into the poor-risk category [1]. Those patients with multiple extrathoracic organ involvement fare even worse and usually have very short 1-year survival if they also present with concurrent symptomatic brain metastases [1]. Front-line conventional chemotherapy using bleomycin, 


\section{Case Reports in Oncology}

Case Rep Oncol 2021;14:1254-1260

\begin{tabular}{l|l}
\hline DOI: 10.1159/000518401 & ( 2021 The Author(s). Published by S. Karger AG, Basel
\end{tabular} www.karger.com/cro

Chou and Tse: Multimodality Therapy for Mediastinal GCT with Extrathoracic Metastases
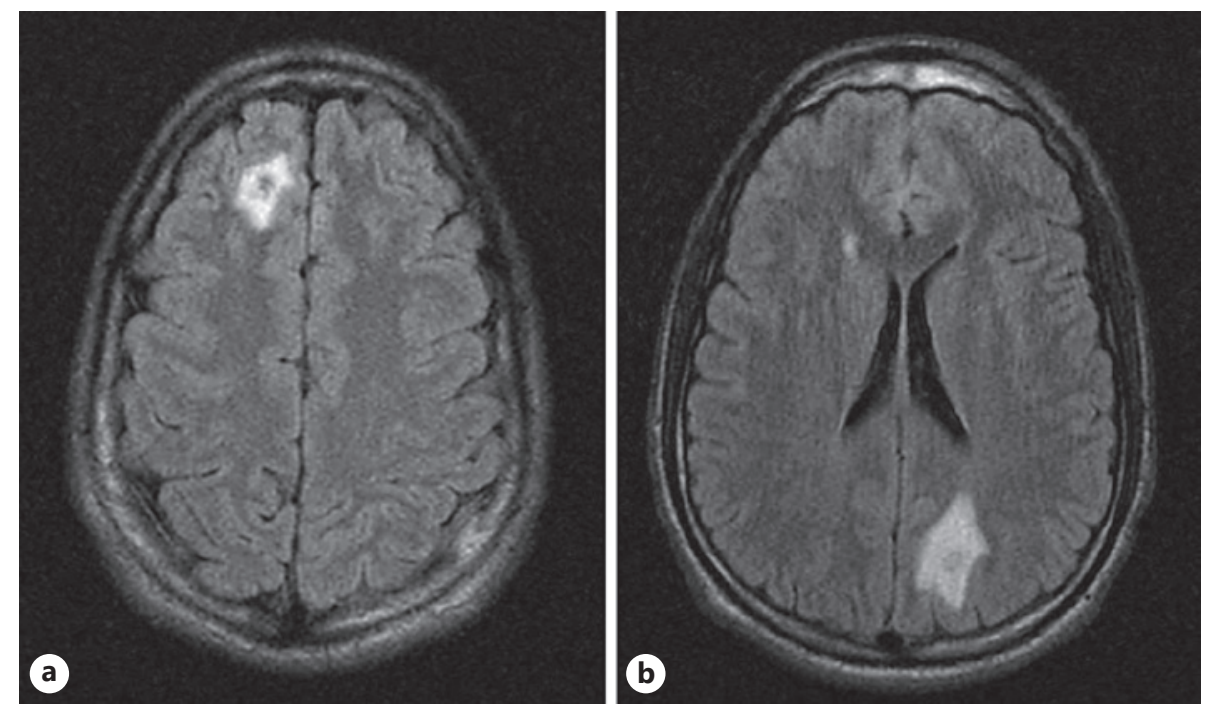

Fig. 1. There were 2 ring-enhancing lesions with a suggestion of hemorrhage in the right frontal (A) and left parietal (B) lobes.

etoposide, and cisplatin (BEP) alone does not offer effective control. The role of upfront highdose chemotherapy with hematopoietic stem cell support (HDCT) in the general population of high-risk NSGCT patients has not been established, and multiple randomized phase 3 trials have not been able to achieve overall survival benefit [2-4]. Subset analyses, however, suggested those whose serum tumor markers (STMs) failed to decline at a satisfactory rate might benefit from front-line HDCT in their clinical outcome [2]. It is not clear whether such benefit could still be demonstrated if HDCT is administered immediately following the completion of standard first-line chemotherapy. It is even more of a challenge to manage a case where an initial response to HDCT was followed by rising STMs shortly afterward. We report the management of a case of primary mediastinal NSGCT with extensive visceral metastases. He survived multimodality therapy and remains disease free.

\section{Case Report}

A 19-year-old male presented with nausea, vomiting, and progressive headaches for 1 month. MRI of the brain showed 2 ring-enhancing lesions in the left parietal lobe and the right frontal lobe with a suggestion of hemorrhage (Fig. 1). CT of the chest, abdomen, and pelvis showed a 9.6- $\mathrm{cm}$ mediastinal mass with both cystic and solid components (Fig. 2A), bilateral lung masses (Fig. 2B), and multiple liver lesions (Fig. 2C). The largest lung lesion measured $6.7 \mathrm{~cm}$. Retroperitoneal lymph nodes were not enlarged. Ultrasound of the scrotum did not reveal any masses. HCG was 1.4 million $\mathrm{mIU} / \mathrm{mL}$ (normal male <2.6), AFP was $38 \mathrm{ng} / \mathrm{mL}$ (normal <3.3), and LDH was $504 \mathrm{IU} / \mathrm{L}$ (normal <250). Biopsy of the mediastinal mass was suggestive but not diagnostic of teratoma. A liver biopsy established a diagnosis of metastatic choriocarcinoma. The syncytiotrophoblasts were positive for HCG.

Initial decision was to start conventional BEP and be evaluated for HDCT during the course of treatment. His STMs were measured weekly during the first 2 cycles of BEP to evaluate the rate of STM decrease and half-life $[2,5]$. It turned out that his STMs did not decline satisfactorily during the first 2 cycles of BEP. At the time of his treatment, it was 

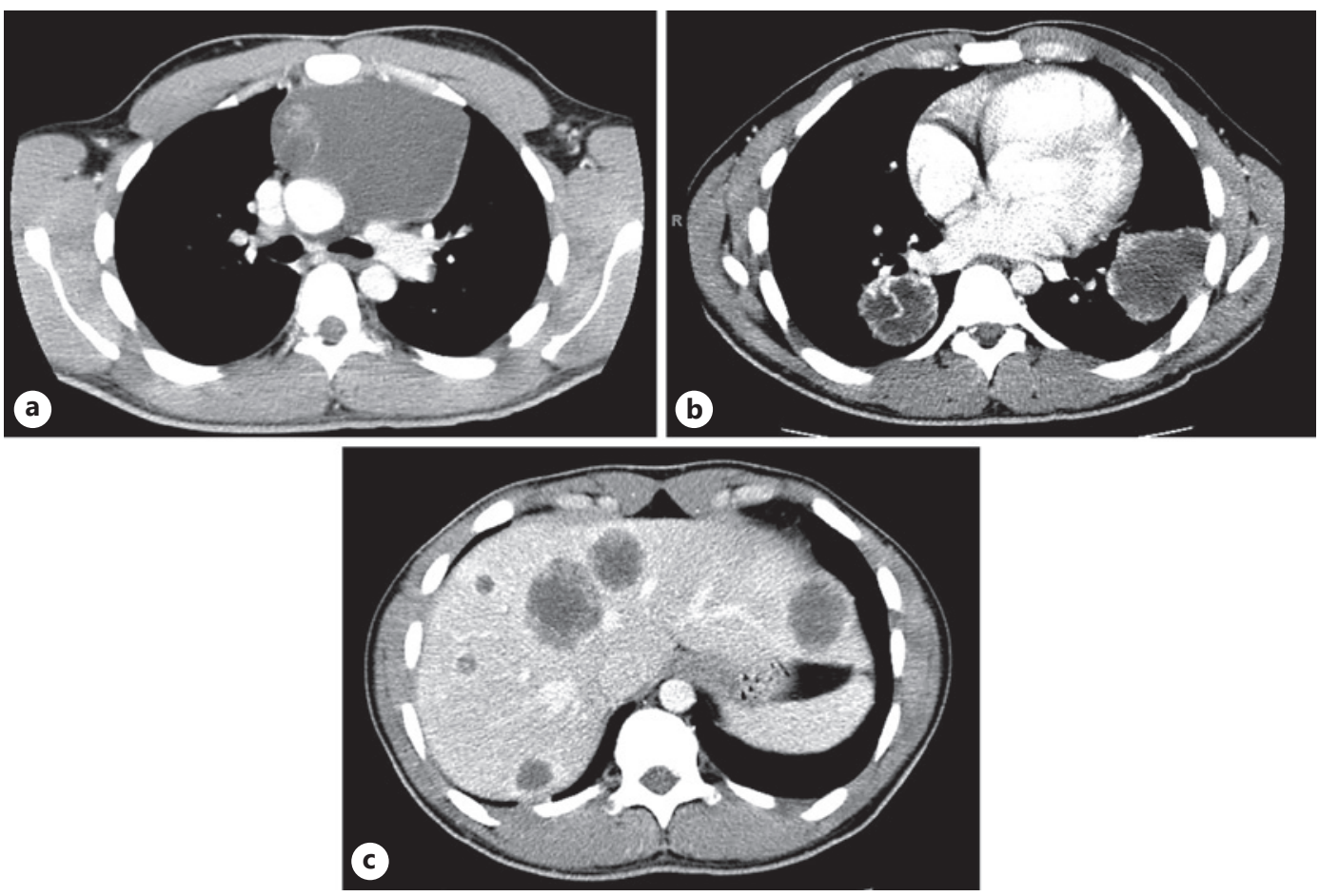

Fig. 2. A A large anterior mediastinal mass which measured up to $9.6 \mathrm{~cm}$ in diameter. This mass contained both cystic and solid components. B Bilateral lung masses at presentation. The largest lung lesion measured $6.7 \mathrm{~cm}$. C There were multiple hypodensities throughout both the right and left lobes of the liver.

reported in a phase 3 trial that a subset of high-risk NSGCT patients, whose STMs had unsatisfactory decline during the first 2 cycles of BEP, benefited from first-line HDCT in 1-year durable complete response and 2-year survival rate [2]. He was referred to bone marrow transplant centers, starting after the first cycle of BEP, for evaluation of upfront HDCT, and it was decided that he needed to finish 4 cycles of BEP. He tolerated BEP almost uneventfully if were not for perforation of the small intestine 4 days after the first cycle of BEP. Postoperative biopsy showed metastatic choriocarcinoma to the jejunum. Following the fourth cycle of BEP, his HCG (375 mIU/mL) and LDH (588 IU/L) were still elevated. His brain lesions were smaller with resolved surrounding edema. However, there were little changes in the size of the mediastinal mass and the visceral metastases (data not shown).

He was sent to a bone marrow transplant center for re-evaluation. Considering that his STMs were still not normalized and there was no detectable response in any of the visceral metastatic lesions, he was accepted for HDCT. During preparation for HDCT, the cytogenetic studies revealed that he had Klinefelter syndrome (47, XXY), which is known to be associated with primary mediastinal NSGCT. He underwent 2 tandem cycles of HDCT started 5 weeks after his last BEP, with complete normalization of AFP and LDH. His HCG level was further reduced to $23 \mathrm{mIU} / \mathrm{mL}$ although not completely normalized (normal male $<2.6$ ). The lung lesions were reduced in size (Fig. 3A). His liver lesions were reduced in both number and size (Fig. 3B). However, the mediastinal mass did not shrink in size. Following HDCT, he received stereotactic radiation to the 2 areas of brain metastases.

Three months following HDCT, his HCG gradually climbed to $600 \mathrm{mIU} / \mathrm{mL}$, and the CT scan showed that the mediastinal mass was increased in size. Although there was improvement in bilateral lung metastases following HDCT, the largest residual lung metastasis was $>3.0 \mathrm{~cm}$. With a rapid increase of STMs soon after HDCT, salvage chemotherapy would be unlikely to 


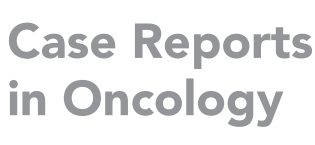

Case Reports

in Oncology
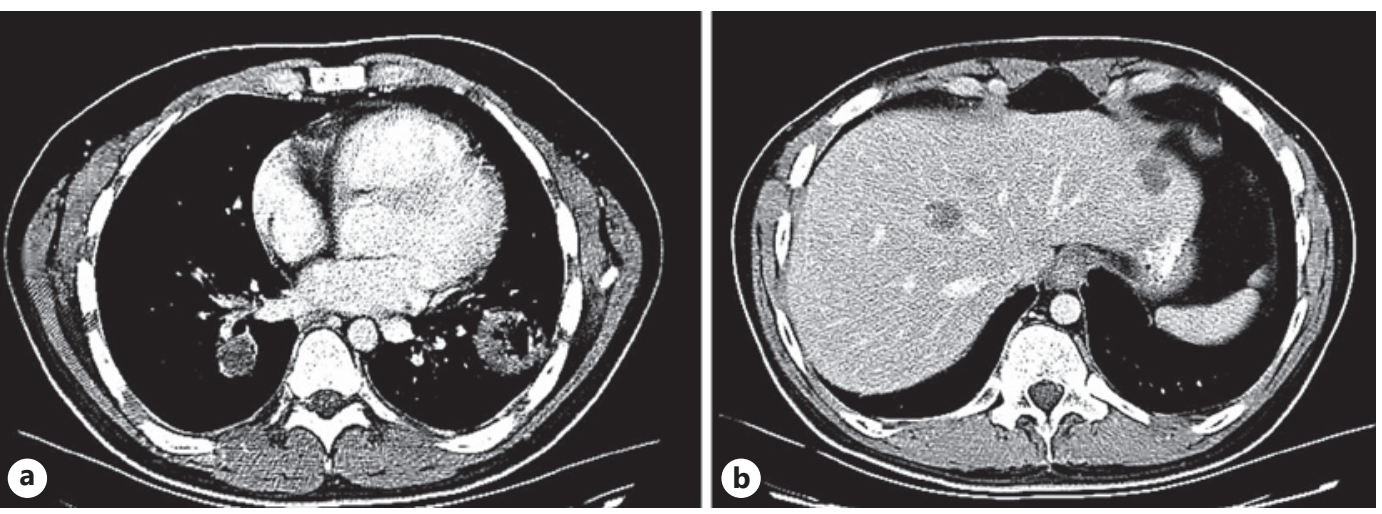

Fig. 3. A Bilateral lung masses were reduced in size following HDCT. B Liver lesions were reduced in both number and size after HDCT.

offer adequate control in this scenario. It was a dire situation, and the outcome of doing nothing was apparently hard to accept for this young man with a good performance status. Surgical resection of the mediastinal germ cell tumor was then entertained. Large residual lung metastases were of concern as they might harbor viable tumor cells. A decision was then reached to perform resection of the mediastinal mass and pulmonary metastasectomy [6-9]. Postoperative pathology confirmed that he had a $10-\mathrm{cm}$ well-circumscribed, mediastinal teratoma with no evidence of choriocarcinoma. The residual lung metastases were consistent with necrotic tissues with no detectable viable malignant cells. His serum HCG levels dropped promptly to $28 \mathrm{mIU} / \mathrm{mL} 1$ week after surgery from a preoperative level of $600 \mathrm{mIU} / \mathrm{mL}$. He received additional 2 cycles of albumin-bound paclitaxel and gemcitabine [10] for fear of potential viable tumor cells in such a bulky tumor specimen and elevated HCG levels prior to surgery in the presence of a growing mediastinal mass. His HCG was $<0.1 \mathrm{mIU} / \mathrm{mL}$ after the completion of the 2 cycles of chemotherapy and has remained normal afterward. His follow-up CT scans showed that his liver lesions continued to shrink. He has remained NED for $>10$ years.

\section{Discussion}

Two main issues from this complicated case merit discussion. The first is the role of HDCT. Several randomized trials failed to demonstrate a survival benefit for upfront HDCT following initial conventional chemotherapy in poor-risk NSGCT [2-4]. However, in the US intergroup study, including 58 cases of primary mediastinal NSGCT [2], it was reported that those with unsatisfactory STM decline during the initial BEP treatment benefited from subsequent HDCT in both 1-year durable CR and 2-year survival rate compared to those treated with BEP alone in a subset analysis. It has been known that the rate of STM decline is an independent prognostic factor to predict response to front-line chemotherapy and survival [5]. It was hypothesized that HDCT probably overcame drug resistance in those whose STM failed to decrease optimally. However, the upfront HDCT in all these trials was designed to be given to eligible participants in the experimental arm following 2 cycles of conventional chemotherapy [2-4], a trial design to balance 4 cycles of conventional chemotherapy in the control arm. It is in contrast to our case where HDCT was started following 4 cycles of BEP although the suboptimal decline of his STMs during the initial BEP probably predicted an unfavorable outcome with conventional chemotherapy. The refractoriness 
was finally established at the completion of BEP based on both dynamic changes of STMs and unchanged number and size of visceral metastases. Such an outcome portended an impending poor prognosis, and it was thus decided to adopt HDCT, in a hope to overcome drug resistance in our case as in the trials using upfront HDAC. In fact, only after HDCT did his lung and liver metastases reduce in number and/or size. Long-term follow-up in this case further proves that high-dose chemotherapy contributed to complete sterilization of all the liver metastases. Our case lends support to the observation that HDCT has a role in certain selective individuals with primary mediastinal NSGCT who could be identified as being refractory to first-line BEP. Nevertheless, it would be ideal for such patients to be enrolled in clinical trials where available.

Another issue is how to deal with rising STMs and persistent presence or regrowth of primary mediastinal tumor following HDCT. Unlike gonadal germ cell tumor, primary mediastinal NSGCT is rare and accounts for only 5\% of total GCT. Consequently, there are much fewer reports about the management of refractory cases. It is known that primary mediastinal NSGCT is classified in the IGCCCG-defined poor prognosis subgroup [1], and its cure rate is inferior to that of testicular GCT, likely due to different biological behavior [11]. There is a high likelihood that primary mediastinal NSGCT tends to have residual disease refractory to first-line chemotherapy [12]. Following first-line chemotherapy, surgical resection of the primary site is recommended irrespective of STM status [13] as salvage chemotherapy offers little disease control [14] unlike testicular GCT [13]. In primary mediastinal NSGCT patients who are refractory to first-line chemotherapy, there is a large case series regarding the role of salvage surgical resection of residual disease upon rising STMs following front-line conventional chemotherapy [15]. In this single institution report, 35 patients were identified with rising STMs following standard cisplatin-based chemotherapy based on a retrospective review of 158 consecutive cases of primary mediastinal NSGCT from 1982 to 2007. When initial chemotherapy was started, 24 out of the 35 patients had disease confined to the mediastinum, 10 had lung involvement, and 3 had liver or brain involvement. Surgery involved a complete en bloc removal of the residual mass, thymus, and surrounding involved structures [16]. In the 35 cases who underwent surgical resection, 7 patients (20\%), including 2 patients with lung involvement, were still alive at the time of report, with a median follow-up of 5 years. Nevertheless, none of those patients with liver or brain metastases survived following surgery. Another single-institution review over 27 years identified 21 cases of primary mediastinal NSCGC, who had persistently elevated STMs following front-line cisplatin-based chemotherapy and underwent resection of residual disease. That approach resulted in long time survival in 8 patients with a 5 -year survival of $36 \%$ in this selective population [17]. Those with disease confined to the mediastinum achieved better survival than lung/pleural involvement. Again, neither of the 2 patients with liver metastases survived $>6$ months following surgery. Incomplete resection in 3 patients led to demise. Apart from the impact of disease extent, other factors such as pulmonary toxicity from bleomycin and availability of surgical expertise also affected outcome. Apparently, surgical resection of residual disease among those who were refractory to first-line chemotherapy appeared to save lives in some while represented a desperation procedure to others. Although our case did have unfavorable prognostic factors such as both liver and brain metastases, he did achieve radiographic response in all visceral metastatic lesions following HDCT. It was worthwhile to take surgical approach in this desperate situation. The eventual normalization of STMs following salvage surgical resection and long-term follow-up of this case suggested that surgical approach may be applicable to highly selective patients with primary mediastinal NSGCT. Finally, for the purpose of optimal disease control, stereotactic radiation to brain metastases should be considered to maximize treatment benefit with a goal to cure every patient with germ cell tumor [18].

\section{Karger'}




\section{Case Reports in Oncology}

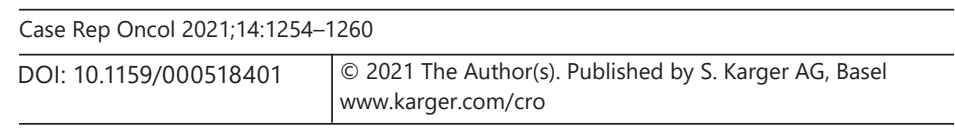

Chou and Tse: Multimodality Therapy for Mediastinal GCT with Extrathoracic Metastases

\section{Conclusion}

This complicated case illustrates the importance of multimodality therapy. HDCT following BEP offered clinical benefit in this high-risk primary mediastinal NSGCT patient who was refractory to conventional chemotherapy as evidenced by initial unsatisfactory STM decline and little response in visceral metastases following the completion of BEP. Resection of residual mediastinal NSGCT and pulmonary metastasectomy offered curability in spite of rising STMs and extensive extrathoracic diseases. Stereotactic brain radiation also contributed to long-term disease control.

\section{Acknowledgments}

The authors wish to thank Drs. R. Spielberger and M. Girvigian for their assistance in high-dose chemotherapy with stem cell support and stereotactic radiation.

\section{Statement of Ethics}

We have obtained written informed consent from the patient to publish this case report and the accompanying images. Approval from an ethics committee is not required as this report is not about clinical research or study. It is about day-to-day management of a cancer patient based on either standard treatment protocol or published data with relevant references provided.

\section{Conflict of Interest Statement}

The authors declared no conflicts of interest.

\section{Funding Sources}

This work was not supported by any source of funding.

\section{Author Contributions}

Jia-Ling Chou was involved in manuscript writing and literature review. David Tse was involved in editing and proof reading. Both authors were involved in the patient management.

\section{Data Availability Statement}

All data generated or analyzed in this case report are included in this article and/or its figures. Further enquiries can be directed to the corresponding author.

\section{Karger' ${ }^{\prime \prime}$}




\section{References}

1 International Germ-Cell Cancer Collaborative Group. International germ-cell consensus classification: a prognostic factor-based staging system for metastatic germ-cell cancers. J Clin Oncol. 1997 Feb;15(2):594-603.

2 Motzer RJ, Nichols CJ, Margolin KA, Bacik J, Richardson PG, Vogelzang NJ, et al. Phase III randomized trial of conventional-dose chemotherapy with or without high-dose chemotherapy and autologous hematopoietic stem-cell rescue as first-line treatment for patients with poor-prognosis metastatic germ cell tumors. J Clin Oncol. 2007 Jan;25(3):247-56.

3 Droz JP, Kramar A, Biron P, Pico JL, Kerbrat P, Pény J, et al. Failure of high-dose cyclophosphamide and etoposide combined with double-dose cisplatin and bone marrow support in patients with high-volume metastatic nonseminomatous germ-cell tumors: mature results of a randomized trial. Eur Urol. 2007 Mar;51(3): 739-46.

4 Daugaard G, Skoneczna I, Aass N, De Wit R, De Santis M, Dumez H, et al. A randomized phase III study comparing standard dose BEP with sequential high-dose cisplatin, etoposide, and ifosfamide (VIP) plus stem-cell support in males with poor-prognosis germ-cell cancer. An intergroup study of EORTC, GTCSG, and grupo germinal (EORTC 30974). Ann Oncol. 2011 May;22(5):1054-61.

5 Mazumdar M, Bajorin DF, Bacik J, Higgins G, Motzer RJ, Bosl GJ. Predicting outcome to chemotherapy in patients with germ cell tumors: the value of the rate of decline of human chorionic gonadotrophin and alphafetoprotein during therapy. J Clin Oncol. 2001 May;19(9):2534-41.

6 Vulky J, Bains M, Bacik J, Higgins G, Bajorin DF, Mazumdar M, et al. Role of postchemotherapy adjunctive surgery in the management of patients with nonseminoma arising from the mediastinum. J Clin Oncol. 2001 Feb;19(3):682-8.

7 Rick O, Bokemeyer C, Weinknecht S, Schirren J, Pottek T, Hartmann JT, et al. Residual tumor resection after high-dose chemotherapy in patients with relapsed or refractory germ cell cancer. J Clin Oncol. 2004 Sep; 22(18):3713-9.

8 McGuire MS, Rabbani F, Mohseni H, Bains M, Motzer R, Sheinfeld J. The role of thoracotomy in managing postchemotherapy residual thoracic masses in patients with nonseminomatous germ cell tumours. BJU Int. 2003 Apr;91(6):469-73.

9 Pfannschmidt J, Hoffmann H, Dienemann H. Thoracic metastasectomy for nonseminomatous germ cell tumors. J Thorac Oncol. 2010 Jun;5(6 Suppl 2):S182-6.

10 Einhorn LH, Brames MJ, Juliar B, Williams SD. Phase II study of paclitaxel plus gemcitabine salvage chemotherapy for germ cell tumors after progression following high-dose chemotherapy with tandem transplant. J Clin Oncol. 2007 Feb;25(5):513-6.

11 Bokemeyer C, Nichols CR, Droz JP, Schmoll HJ, Horwich A, Gerl A, et al. Extragonadal germ cell tumors of the mediastinum and retroperitoneum: results from an international analysis. J Clin Oncol. 2002 Apr;20(7):1864-73.

12 Ganjoo KN, Rieger KM, Kesler KA, Sharma M, Heilman DK, Einhorn LH, et al. Results of modern therapy for patients with mediastinal nonseminomatous germ cell tumors. Cancer. 2000 Nov;88(5):1051-6.

13 Honecker F, Aparicio J, Berney D, Beyer J, Bokemeyer C, Cathomas R, et al. ESMO consensus conference on testicular germ cell cancer: diagnosis, treatment and follow-up. Ann Oncol. 2018 Aug;29(8):1658-86.

14 Hartmann JT, Einhorn L, Nichols CR, Droz JP, Horwich A, Gerl A, et al. Second-line chemotherapy in patients with relapsed extragonadal nonseminomatous germ cell tumors: results of an international multicenter analysis. J Clin Oncol. 2001 Mar;19(6):1641-8.

15 Radaideh SM, Cook VC, KeslerEinhorn KALH, Einhorn LH. Outcome following resection for patients with primary mediastinal nonseminomatous germ-cell tumors and rising serum tumor markers post-chemotherapy. Ann Oncol. 2010 Apr;21(4):804-7.

16 Kesler KA. Technique of mediastinal germ cell tumor resection. Oper Tech Thorac Cardiovasc Surg. 2009; 14(1):55-65.

17 De Latour B, Fadel E, Mercier O, Mussot S, Fabre D, Fizazi K, et al. Surgical outcomes in patients with primary mediastinal non-seminomatous germ cell tumours and elevated post-chemotherapy serum tumour markers. Eur J Cardiothorac Surg. 2012 Jan;42(1):66-71.

18 Bosl G. Recurrent germ cell tumors: let's try to cure them all. J Onc Practice. 2016 May;12(5):445-8. 